\title{
Synthetic $\theta$-Defensin Antibacterial Peptide as a Highly Efficient Nonviral Vector for Redox-Responsive miRNA Delivery
}

\author{
Meng Yu, Jin Yan, Wangxiao He,* Chenyu Li, Peter X. Ma, and Bo Lei*
}

Synthetic cationic vectors have shown great promise for nonviral gene delivery. However, their cytotoxicity and low efficiency impose great restrictions on clinic applications. To push through this limitation, humanized peptides or proteins with cationic biocompatibility as well as biodegradation would be an excellent candidate. Herein, for the first time, we describe how an arginine-rich humanized antimicrobial cyclopeptide, $\theta$-defensin, can be used as a synthetic cationic vector to load and deliver miRNA into bone mesenchymal stem cells with high efficiency and ultralow cytotoxicity, surpassing the efficiency of the commercial polyethylenimine (25 kD) and Lipofectamine 3000 . To note, $\theta$-defensin can redox-responsively release the loaded miRNA through a structural change: in extracellular oxidative environment, $\theta$-defensin has large $\beta$-sheet structures stabilized by three disulfide linkages, and this special structure enables highly efficient delivery of miRNA by passing through cell membranes; in intracellular environment, redoxresponsive disulfide linkages are broken and the tight $\beta$-sheet structures are destroyed, so that the miRNA can be released. Our results suggest that synthetic $\theta$-defensin peptides are a new class of nonviral gene vectors and this study may also provide a promising strategy to design smart-responsive gene vectors with high efficiency and minimal toxicity.

especially miRNA transfection and delivery need to be improved. The transfection or delivery of miRNA to target cells could efficiently treat various human diseases such as cancers and cardiovascular diseases, but has been limited by the low membrane permeability and poor serum stability of miRNAs. ${ }^{[4-6]}$ Although cationic polymers and liposomes are widely investigated as nonviral vectors to overcome the intrinsic weaknesses of nucleotides, their miRNAs delivery was always unsatisfactory due to their cytotoxicity and uncontrollability. ${ }^{[7-9]}$ To overcome these limitations, it is very necessary to develop next generation nonviral vectors with high miRNA delivery efficiency, minimal cytotoxicity, and smart responsive release. ${ }^{[10]}$

Theta defensin ( $\theta$-defensin), a family of arginine-rich humanized antimicrobial cyclopeptides with large $\beta$-sheet structures stabilized by disulfide linkages and connected by turns, is well known for its antivirus and antibacterial activity via destabilizing the microprotein by the high positive charge density of defensin. ${ }^{[11-13]}$

MicroRNAs (miRNAs), a class of short noncoding RNAs (18-25 nt), play an important role in regulating various biological processes through modulating post-transcriptional gene expression of gene targets, including cell proliferation, differentiation, and apoptosis. ${ }^{[1-3]}$ In biologically relevant cell models and cell therapy, the existing molecular and cellular techniques,
Previous studies have shown that $\theta$-defensin is nontoxic and nonimmunogenic in vitro and in vivo, suggesting its great biocompatibility. ${ }^{[14-16]} \theta$-defensin can also suppress the production of proinflammatory cytokines and modulate the immune response, indicating its potential in disease therapy. ${ }^{[17-19]}$ Additionally, the Arg-Gly-X (RGX) sequence-contained structure
Dr. M. Yu, Prof. B. Lei

Frontier Institute of Science and Technology

State Key Laboratory for Mechanical Behavior of Materials

Xi'an Jiaotong University

Xi'an 710054, China

E-mail: rayboo@xjtu.edu.cn

Dr. J. Yan, Dr. W. He

Center for Translational Medicine

Key Laboratory of Biomedical Information Engineering

of Ministry of Education

School of Life Science and Technology and Frontier Institute

of Science and Technology

Xi'an Jiaotong University

Xi'an 710049, China

E-mail: hewangxiao@stu.xjtu.edu.cn

\author{
Dr. C. Li \\ School of Science \\ Xi'an Jiaotong University \\ Xi'an 710054, China \\ Prof. P. X. Ma \\ Department of Biologic and Materials Sciences \\ Department of Biomedical Engineering \\ Macromolecular Science and Engineering Center \\ Department of Materials Science and Engineering \\ University of Michigan \\ Ann Arbor, MI 48109, USA
}

DOI: 10.1002/adbi.201700001 
in $\theta$-defensin enables the potent binding ability for integrin which is highly expressed at the surface of bone mesenchymal stem cells (BMSCs). ${ }^{[20]}$ As progenitor cells, BMSCs can differentiate into various tissue cells and have exhibited promising applications in tissue regeneration and disease therapy. ${ }^{[21]}$ After binding to the integrin of BMSCs and internalization into the cells, the reducing cytoplasm breaks the disulfide linkages of $\theta$-defensin and reduces its positive charge density. ${ }^{[22]}$ Following this, the loaded genes will be redox-responsively released and the reducing $\theta$-defensin can be biodegraded into amino acids via enzymolysis. These unique properties endow $\theta$-defensin with promising potential for highly efficient miRNA delivery.

Herein, for the first time, we present the performance of synthetic $\theta$-defensin peptides as highly efficient nonviral vectors for miRNA-5106 (miR-5106) delivery to BMSCs for regulating their osteoblastic differentiation to settle for bone tissue engineering application. For embodying the excellent transfection capacity of $\theta$-defensin, Lipofectamine 3000, the best commercial nonviral vector known as having the most superior efficiency and lowest toxicity was chosen to be compared with $\theta$-defensin. Meanwhile, PEI 25K, the most common commercial vector for DNA or RNA with reliable outcomes, was used as positive control in this work. In a previous study, miR-5106 was identified from BMSCs and showed a good performance to enhance osteoblastic differentiation of BMSCs. ${ }^{[23]} \mathrm{A}$ further study showed that miR-5106 could significantly enhance osteogenic gene expressions (Runx2, Sp7) through targeting Gsk3a, which was proved by quantitative real-time polymerase chain reaction (qRT-PCR), dual-luciferase vector analysis and western blot experiment (Figure S1, Supporting Information). The improved osteogenic differentiation by the downregulation of Gsk3a was also seen in previous references. ${ }^{[24,25]}$ As shown in Scheme 1, the 18-residue $\theta$-defensin peptides (RC101, an artificial mutant from the natural Theta-Defensin Retrocyclin) including three arginine, a lysine, and three disulfide bond, was synthesized by a solid phase peptide synthesis (SPSS) and native chemical ligation (NCL). The RC101 with a high positive charge density and good cell-penetrating ability could efficiently condense miR-5106 and deliver it into cells for regulating target gene expressions. In the intracellular reductive environment, the three disulfide bonds in RC101 would be broken, so that dense $\beta$-sheet structure broke down and changed to an incompact loop. And this structure change of RC101 would lead to the decrease of positive charge density, which could reduce the adsorption of the electronegative miR-5106 and enable the redox-responsive controlled release of miR-5106 (Scheme 1). After that, miR-5106 may incorporate into RNA induced silencing complex (RISC), mediate Gsk3a mRNA translational repression, and induce osteogenic regulator gene Runx2, and Sp7 expression which could enhance BMSC osteoblastic differentiation (Scheme 1).

To validate this concept, a backbone ribbon model of RC101 and its reduced form, were first made by templating its sequence on the structure of protegrin-1 (PG-1), and cyclizing it (Figure 1A). ${ }^{[26]}$ The resulting structure was annealed by the molecular dynamics as well as energy minimized, and further verified by circular dichroism spectrum (Figure 1B). These results confirmed the dense $\beta$-sheet structure of RC101, and this structure can be changed to a flexible loop at the reduced form. Moreover, the native and reduced structure of RC101 were confirmed by high performance liquid chromatography (HPLC) and electrospray ionization mass spectrometry (ESI-MS), and the increase of molecular weight from 1890.2 to $1896.4 \mathrm{Da}$ indicated the breaking of three disulfide bonds after reduction (Figure 1C,D). The dynamic simulation clearly showed that RC101 demonstrated significantly high positive charge density compared with the reduced RC101 (Figure S2, Supporting Information). RC101 possessed a zeta potential of $42.3 \mathrm{mV}$ and this value was significantly decreased to $0.08 \mathrm{mV}$ after reduction (Figure 1E). These results suggested that in extracellular oxidative environment, $\theta$-defensin has large $\beta$-sheet structures stabilized by three disulfide linkages, and this arginine-rich compact structure has the high positive charge density to adsorb miRNA; in intracellular environment, redox-responsive disulfide linkages were broken and the tight $\beta$-sheet structures were destroyed, so the positive charge density sharped fall (reduced RC101) and miRNA can be released. The structure of RC101 is well suited for delivering miRNA compared to reduced RC101. To validate the effectiveness of RC101 in delivering miRNA, we detect the binding ability and stability of RC101-miRNA (Figure 1; Figures S3 and S4, Supporting Information). To demonstrate the promising potential of RC101 for miRNA delivery, the RC101-mediated miR-5106 transfection efficiency in BMSCs was evaluated, using commercial transfection reagents PEI $25 \mathrm{~K}$ and Lipo as controls (Figure 2; Figures S5-S10, Supporting Information) by viability test, confocal microscopy, real-time PCR analysis, and von Kossa stain.

After incubating with miR-5106, the representative RC101@ miR-5106 and reduced RC101@miR-5106 nanocomplexes with a size of 50-70 nm were formed (Figure S3, Supporting Information). The gel electrophoresis analysis showed that the miR-5106 was efficiently condensed by RC101 at a weight ratio of 30:1, which was comparable to PEI 25K (Figure S4A, Supporting Information). Due to the strong positive charge density, RC101 showed an ₹twofold high miR-5106 loading ability compared with the reduced RC101, as well as significantly high binding capacity relative to commercial PEI $25 \mathrm{~K}$ at the same mass (Figure $1 \mathrm{~F}$ ). In the presence of polyanion (heparin), RC101 still exhibited almost two times higher miR-5106 binding stability than PEI 25K, and over 14-fold higher compared with commercial Lipo and reduced RC101 (Figure 1G; Figure S4B, Supporting Information). Additionally, the serum stability test clearly showed that RC101 completely protected miR-5106 against the enzymolysis over $3 \mathrm{~h}$, whereas the naked miR-5106 as well as Lipo@ miRNA were completely digested during $3 \mathrm{~h}$ with a half-life time of 0.26 and $0.27 \mathrm{~h}$, respectively (Figure 1H; Figure S4C, Supporting Information). These results unequivocally demonstrate that RC101 may be an excellent nonviral vector for miRNA delivery, based on its high efficient miRNA loading and protecting miRNA against serum degradation and redox-responsive controlled release of miRNAs.

The cytotoxicity of PEI-based polymers and Lipo is one of the main obstacles to limit their successful clinical gene therapy applications. Therefore, it is necessary to evaluate the cytotoxicity of nonvirial vectors before their applications. Here, RC101 with different concentrations (30-240 $\mu \mathrm{g} \mathrm{mL}^{-1}$ ) showed 

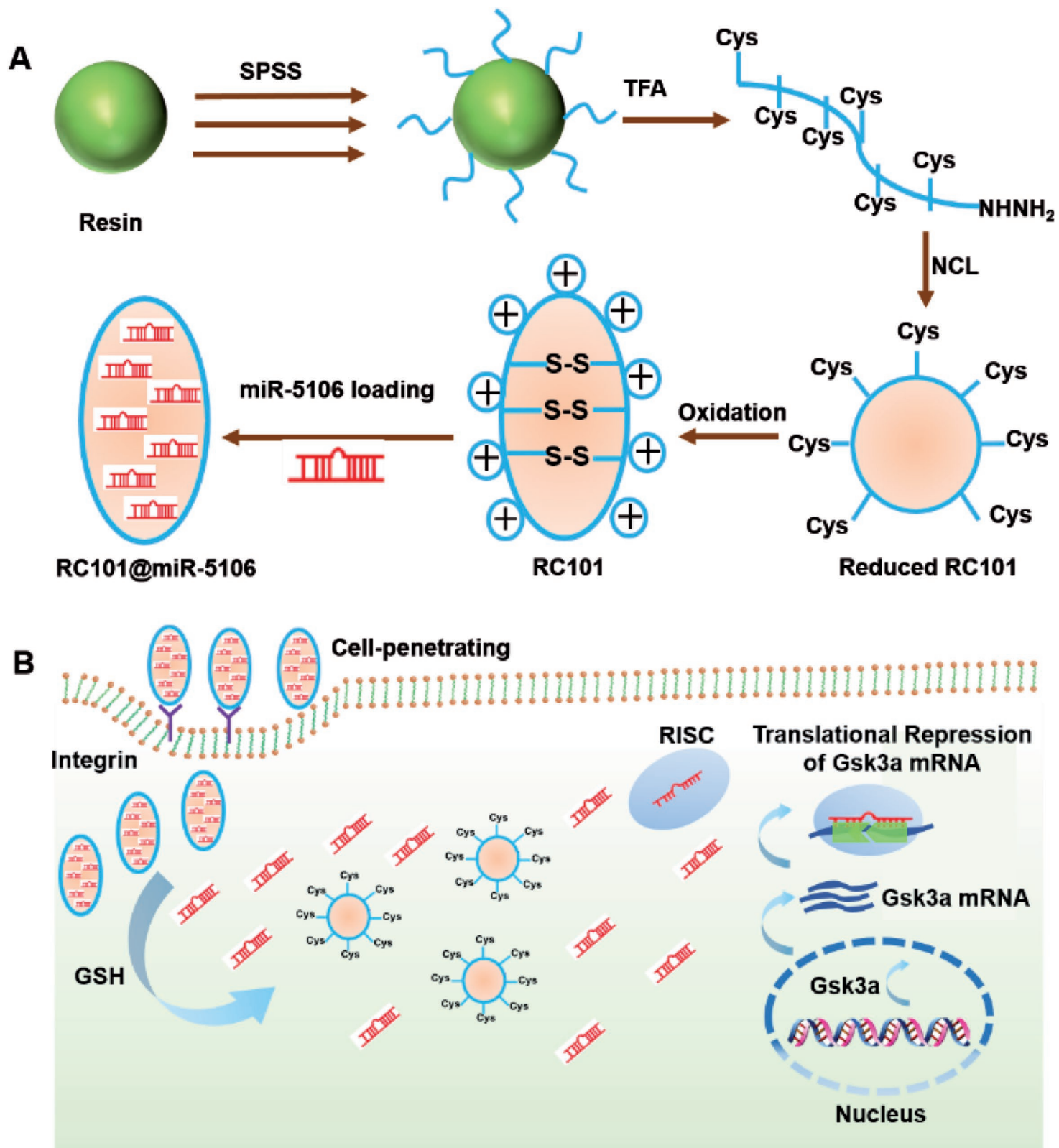

Scheme 1. Schematic illustration of synthesized $\theta$-defensin peptides (RC101) for miRNA delivery. A) Reduced RC101 was synthesized by a solid phase peptide synthesis (SPSS) and native chemical ligation (NCL). After the oxidation of disulfide bonds, the miR-5106 can be loaded efficiently on the surface of the oxidative form of reduced RC101; B) RC101 with a high positive charge density and good cell-penetrating ability could efficiently condense miR-5106 and deliver it into BMSCs for regulating target gene expressions. In the intracellular reductive environment (GSH), the three disulfide bonds in $\mathrm{RC} 101$ would be broken, which could reduce the adsorption of the electronegative miR-5106 and enable the redox-responsive controlled release of miR-5106. Over-expression of miR-5106 could downregulate Gsk3a expression, which may result in osteogenic differentiation.

a significantly high BMSCs viability and negligible cytotoxicity, while the commercial liposome transfection reagents Lipo and PEI 25K exhibited significant cytotoxicity (Figure 1I; Figure S5, Supporting Information). RC101@miR-5106 with different weight ratios presented ultralow cytotoxicity at day 1 and could enhance the cell proliferation after incubation for $5 \mathrm{~d}$ (Figure 1J; Figure S6A, Supporting Information). Additionally, BMSC proliferation was significantly improved by RC101 during further 5 d culture, as compared to PEI 25K and Lipo (Figure S6B, Supporting Information). The ultralow cytotoxicity of RC101 made it highly competitive as efficient nonvirus vectors for miRNA delivery.

The confocal laser scanning microscope images clearly showed that much more miR-5106 was transfected into BMSCs compared with PEI 25K and Lipo after incubation for $48 \mathrm{~h}$ (Figure 2A,B). RC101@miR-5106 transfected cells exhibited around three (two) times higher fluorescent intensity than PEI 25K (Lipo) groups (Figure 2C). The quantitative flow cytometry result also confirmed the significantly high miR-5106 transfection efficiency, as compared to PEI 25K and Lipo (Figure 2B 
A

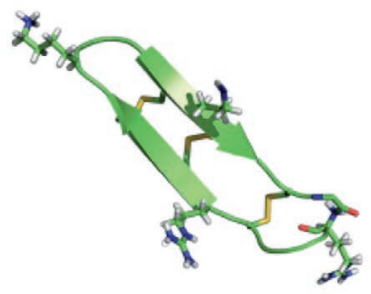

RC101

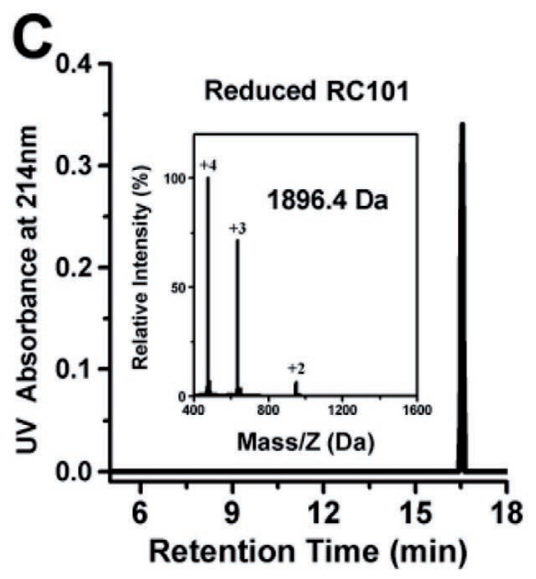

F

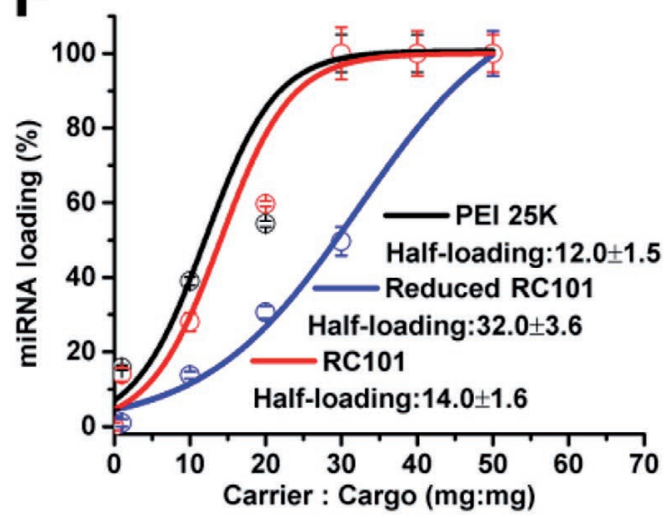

H

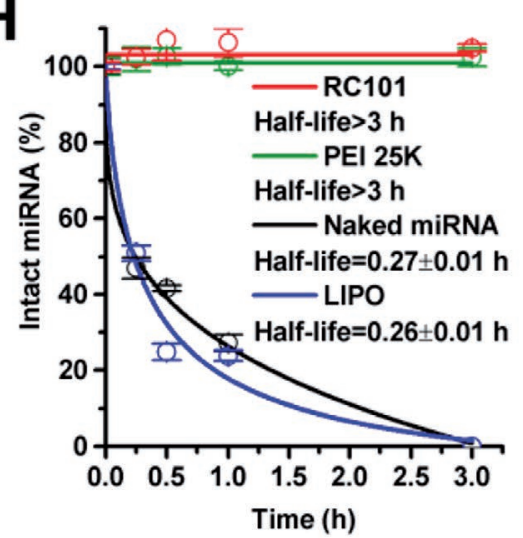

B

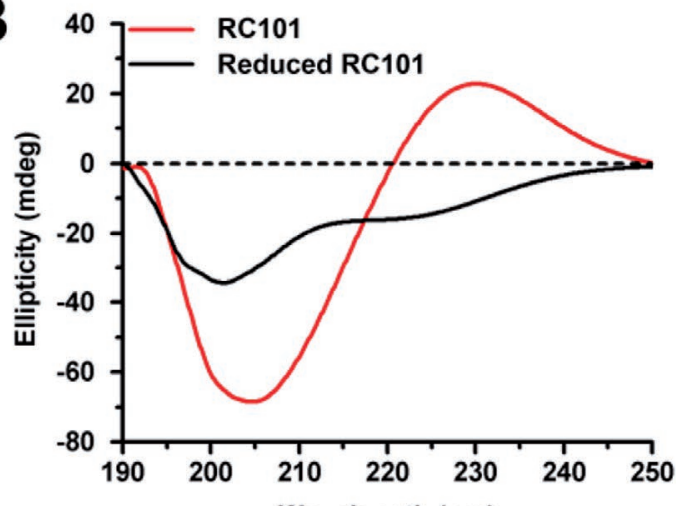

D

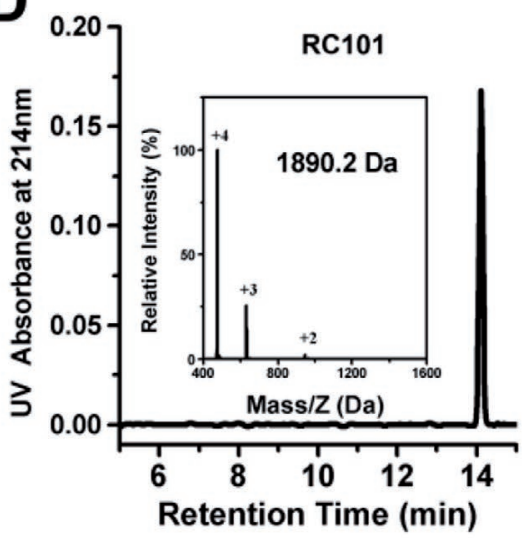

E

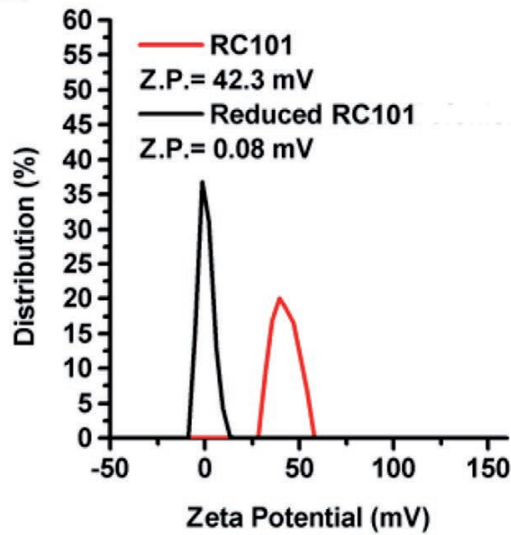

$\mathbf{G}$
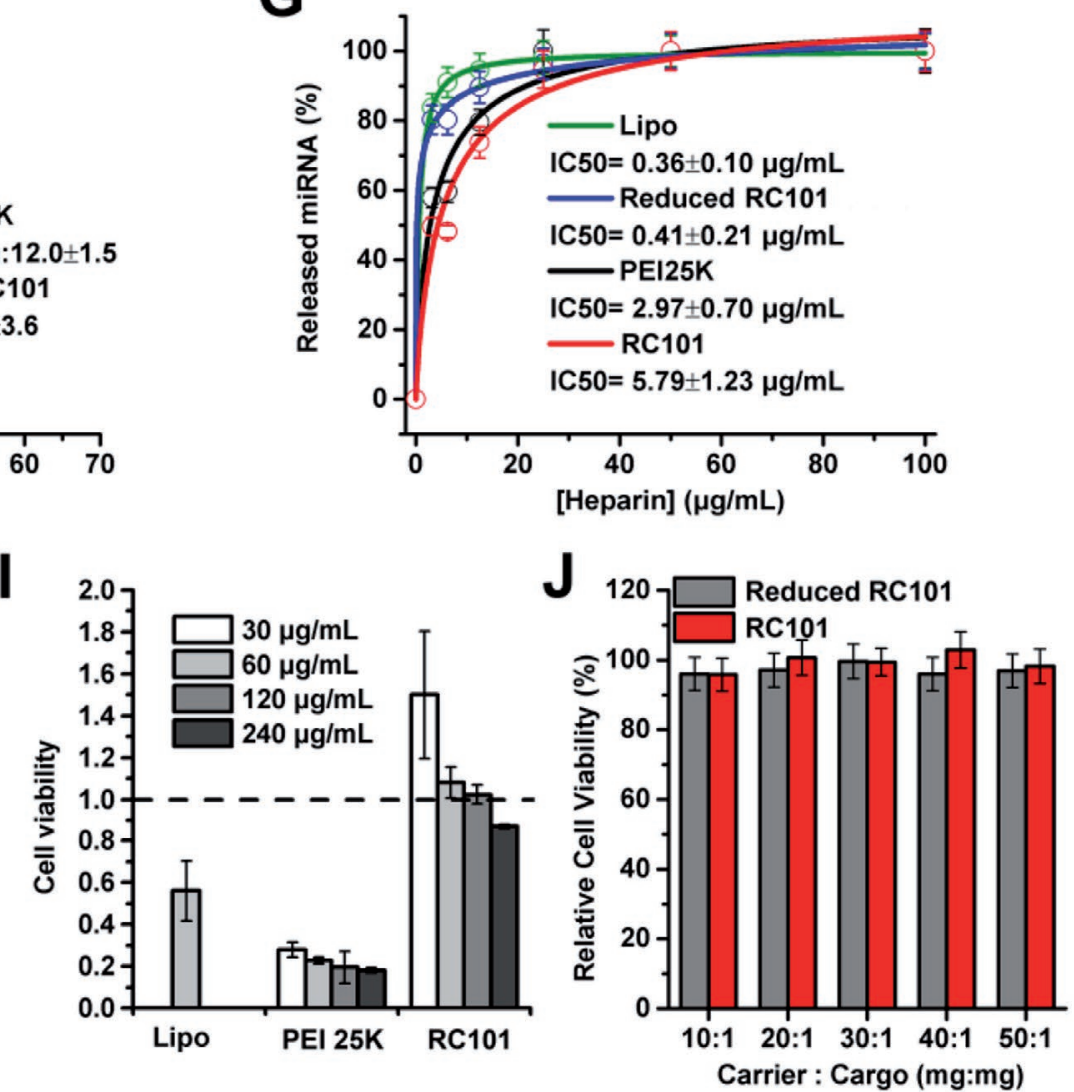


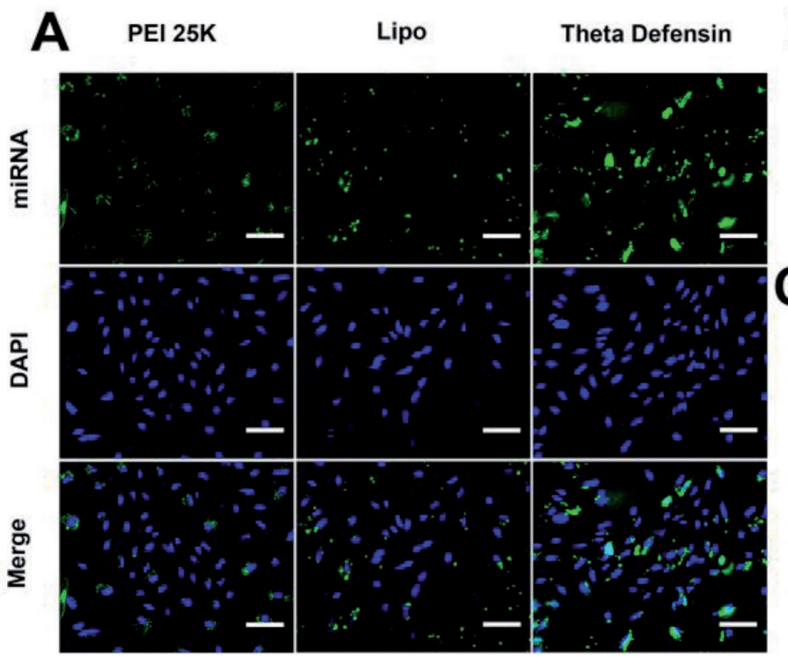

B PEI 25K

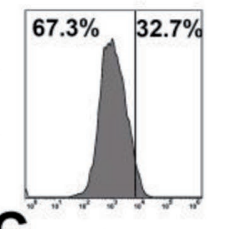

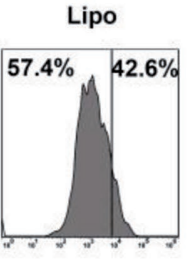

Theta Defensin

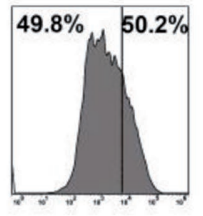

c

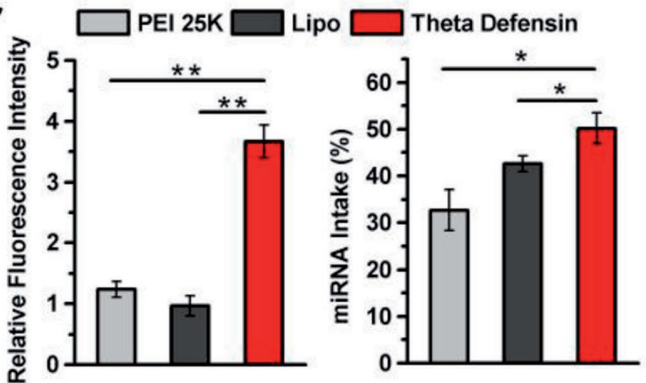

D

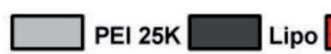

Theta Defensin
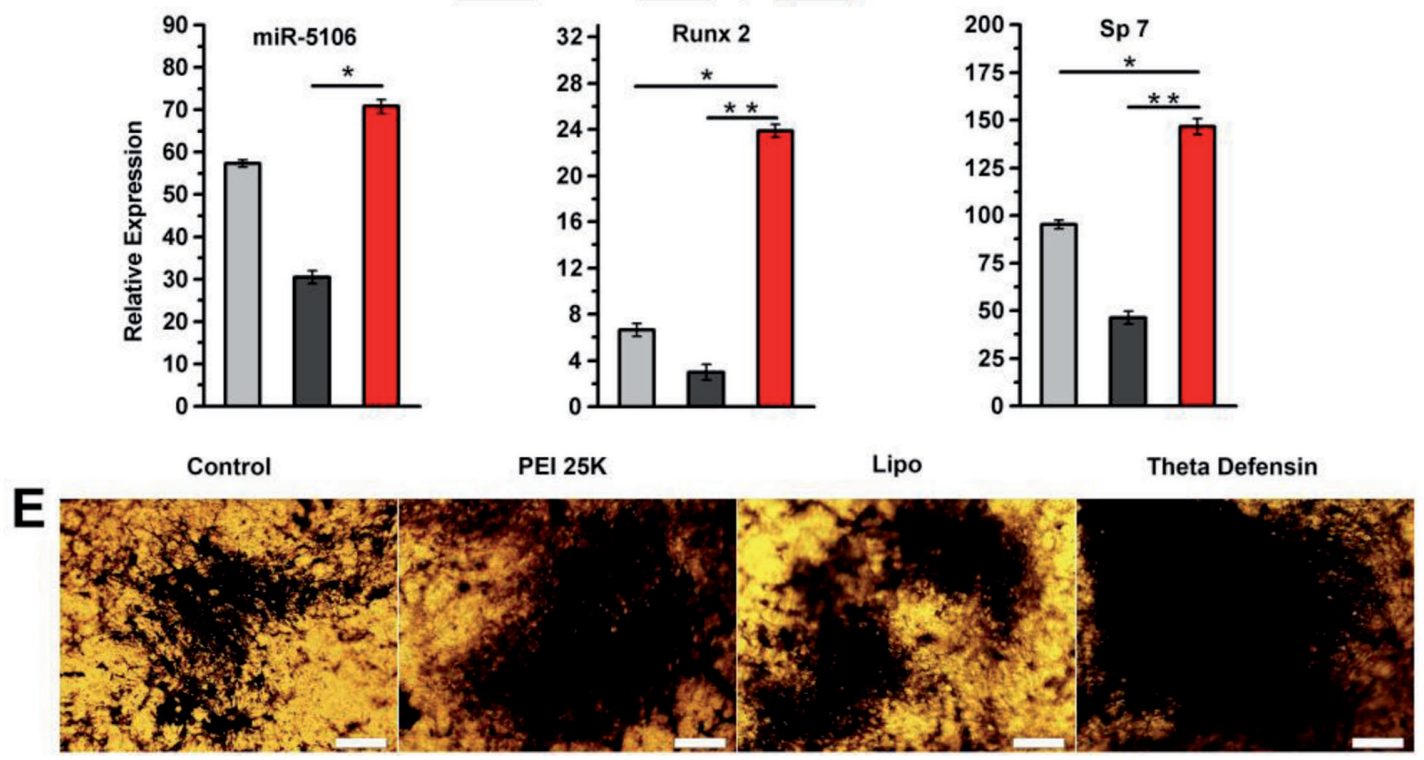

Figure 2. Highly efficient miRNA intracellular delivery, transfection, and gene regulation ability of RC101. A) Confocal laser scanning microscopy pictures of BMSCs with FAM-labeled miRNA transfected by PEI 25K, Lipo, and RC101 (Scale bar $=100 \mu \mathrm{m}$ ); B) flow cytometry analysis in the transfection efficiency of FITC labeled miRNA; C) fluorescence intensity calculated from flow cytometry data and miRNA intake efficiency based on the flow cytometry; B) (* $p<0.05, * * p<0.01$ ); D) RT-PCR analysis of the transfected miR-5160 and the osteogenic differentiation genes (* $p<0.05, * * p<0.01$ ). Normalize by the housekeeping gene, GAPDH; E) osteogenic cellular mineralization evaluation (Von Kossa staining) of BMSCs after induction by miR-5106 mimics delivered by PEI 25K, LIPO, and RC101.

and C). Compared with RC101, the reduced RC101 presented a significantly low miR-5106 transfection ability, indicating the importance of positive charge density in RC101 (Figure S7, Supporting Information). The qRT-PCR analysis normalized by the housekeeping gene, GAPDH, further confirmed that significantly high miR-5106 expression in BMSCs for RC101 group was observed after 3 weeks in comparison with PEI 25K and Lipo (Figure 2D; Figure S8A, Supporting Information).

Figure 1. Structure and properties characterizations of $\theta$-defensin peptides as a miRNA vector. (A) A hypothetical model of the oxidative and reduced form of RC101 made by templating its sequence on the backbone of a similar peptide from porcine neutrophils, protegrin-1 (PDB accession code: 1PG1); (B) CD spectrum of reduced and oxidative RC101, both at $0.5 \mathrm{mg} / \mathrm{ml}$ in PBS at pH 7.4; (C, D) Synthesized reduced (C) and oxidative RC101 (D) were purified by a reverse phase HPLC and their molecular weights were determined by ESIMS; (E) Zeta potentials of reduced RC101 and oxidative RC101 measured in PBS at pH 7.4; (F) MiR-5106 loading ability of PEI 25K, reduced RC101 and oxidative RC101 measured by a direct electrophoretic retardation analysis; (G) MiR-5106 binding affinity of LIPO, PEI 25K, reduced RC101 and oxidative RC101 measured by a heparin competition experiment; (H) Serum stability evaluation of RC101-miR-5106 complexes; (I) Cytotoxicity test of LIPO, PEI 25K and oxidative RC101. (J) Cytotoxicity analysis of RC101-miR-5106 and reduced RC101-miR-5106 complexes. 
In this study, the effectiveness of miR-5106 after transfection into BMSCs by various complexes was verified through investigating the osteoblastic differentiation of BMSCs. After transfecting miR-5106 into BMSCs for 2 and 3 weeks, RC101 group showed a significantly high Sox 9 expression, as compared to PEI 25K, Lipo, and reduced RC101 group (Figure 2D; Figure S8B, Supporting Information). In addition, during the culture periods of 3 weeks, the osteoblastic genes including Runx 2 and Sp 7 were also significantly upregulated in RC101 group in contrast to PEI 25K, Lipo, and reduced RC101 group (Figure 2D; Figure S8C and D, Supporting Information). The transfected reduced RC101@miR-5106 could also enhance the Runx2 expression in BMSCs after 3 weeks (Figure S9, Supporting Information). The enhanced osteoblastic differentiation of BMSCs by RC101@miR-5106 was further confirmed by cellular minerialization staining (Figure 2E). RC101 group demonstrated the significantly strong positive staining (black), as compared with other groups (Figure 2E; Figures S8 and S10, Supporting Information). The significantly high-osteoblastic differentiation performance for RC101@miR-5106 group suggested that the miR-5106 could be released efficiently from RC101 vector. The RC101 also demonstrated the significant antibacterial activity at different concentrations, indicating its potential multifunctional applications (Figure S11, Supporting Information).

In summary, we have demonstrated a novel synthetic $\theta$-defensin peptide, RC101, which possesses several advantages as the next generation gene vector: high biocompatibility and miRNA loading, good serum stability, strong cellular uptake, and transfection efficiency, redox-responsive miRNA controlled release in cells. The RC101@miR-5106 is highly effective to enhance the osteoblastic differentiation of BMSCs compared with commercial transfection reagents PEI 25K and Lipo. Our work first presents that the synthetic $\theta$-defensin peptide is an excellent candidate for highly efficient miRNAs delivery, and may have promising applications in smart gene therapy. This study may also provide a new strategy to develop next generation smart defensin peptide-based gene delivery system, notwithstanding there exist some potential limitations such as DNA transfection and targeting transfection in vivo necessarily to be overcome.

\section{Supporting Information}

Supporting Information is available from the Wiley Online Library or from the author.

\section{Acknowledgements}

M.Y., J.Y., and W.H. contributed equally to this work. We acknowledge the valuable comments of potential reviewers. This work was supported by State Key Laboratory for Mechanical Behavior of Materials (Grant No. 20161801), the Fundamental Research Funds for the Central Universities (Grant No. XJJ2014090), the Natural Science Basic Research Plan in Shaanxi Province of China (Grant No. 2015JQ5165), and National Natural Science Foundation of China (Grant No. 51502237).

\section{Conflict of Interest}

The authors declare no conflict of interest.

\section{Keywords}

defensin peptide, miRNA delivery, redox-responsive, stem cell differentiation

Received: January 10, 2017

Revised: August 24, 2017

Published online: October 24, 2017

[1] Y. Cai, X. Yu, S. Hu, J. Yu, Geno. Prot. Bioinfo. 2009, 7, 147.

[2] V. Ambros, Nature 2004, 431, 350.

[3] L. He, G. J. Hannon, Nat. Rev. Genet. 2004, 5, 522.

[4] J. S. Suh, J. Y. Lee, Y. S. Choi, P. C. Chong, Y. J. Park, Biomaterials 2013, 34, 4347.

[5] N. S. Gandhi, R. K. Tekade, M. B. Chougule, J. Controlled Release 2014, 194, 238.

[6] Y. Chen, D.-Y. Gao, L. Huang, Adv. Drug Deliv. Rev. 2015, 81, 128.

[7] G. Bozzuto, A. Molinari, Int. J. Nanomed. 2015, 10, 975

[8] H. Yin, R. L. Kanasty, A. A. Eltoukhy, A. J. Vegas, J. R. Dorkin, D. G. Anderson, Nat. Rev. Genet. 2014, 15, 541.

[9] R. Arote, T.-H. Kim, Y.-K. Kim, S.-K. Hwang, H.-L. Jiang, H.-H. Song, J.-W. Nah, M.-H. Cho, C.-S. Cho, Biomaterials 2007, 28, 735 .

[10] F. Li, R. I. Mahato, Adv. Drug Deliv. Rev. 2015, 81, v.

[11] S. Yarus, J. M. Rosen, A. M. Cole, G. Diamond, Proc. Natl. Acad. Sci. USA 1996, 93, 14118.

[12] S. Olli, R. Nagaraj, S. R. Motukupally, Antimicrob. Agents Chemother. 2015, 59, 217.

[13] R. I. Lehrer, A. M. Cole, M. E. Selsted, J. Biol. Chem. 2012, 287, 27014.

[14] C. L. Wohlford-Lenane, D. K. Meyerholz, S. Perlman, H. Zhou, D. Tran, M. E. Selsted, P. B. McCray, J. Virol. 2009, 83, 11385.

[15] D. Tran, P. A. Tran, Y.-Q. Tang, J. Yuan, T. Cole, M. E. Selsted, J. Biol. Chem. 2002, 277, 3079.

[16] J. B. Schaal, D. Tran, P. Tran, G. Ösapay, K. Trinh, K. D. Roberts, K. M. Brasky, P. Tongaonkar, A. J. Ouellette, M. E. Selsted, PLoS One 2012, 7, e51337.

[17] R. I. Lehrer, T. Ganz, Curr. Opin. Immunol. 2002, 14, 96.

[18] A. C. Conibear, K. J. Rosengren, N. L. Daly, S. T. Henriques, D. J. Craik, J. Biol. Chem. 2013, 288, 10830.

[19] M. E. Selsted, A. J. Ouellette, Nat. Immunol. 2005, 6, 551.

[20] S. E. D'Souza, M. H. Ginsberg, E. F. Plow, Trends Biochem. Sci. 1991, 16, 246

[21] J. M. Oliveira, M. T. Rodrigues, S. S Silva, P. B. Malafaya, M. E. Gomes, C. A. Viegas, I. R. Dias, J. T. Azevedo, J. F. Mano, R. L. Reis, Biomaterials 2006, 27, 6123.

[22] E. Arnett, S. Seveau, Curr. Pharm. Design 2011, 17, 4254.

[23] M. Yu, B. Lei, C. Gao, J. Yan, P. X. Ma, Nano Res. 2017, 10, 49.

[24] M. K. Ravi, R. G. Anyonya, I. R. Martina, C. N. Michael, Dev. Biol. 2005, 285, 496.

[25] H. K. Nalini, E. O. Jude, Q. Z. Qing, T. Xioayan, L. Min, L. H. David, A. F. Charles, E. Thomas, W. Tao, K. T. Aidas, M. John, S. Masahiko, U. B. Henry, L M. Yanfei, J. Bone Miner. Res. 2006, 21, 910.

[26] S. S. Harwig, A. Waring, H. J. Yang, Y. Cho, L. Tan, R. I. Lehrer, Eur. J. Biochem. 1996, 240, 352 